\title{
A NONLINEAR SEMIGROUP FOR A FUNCTIONAL DIFFERENTIAL EQUATION \\ BY
}

DENNIS W. BREWER ${ }^{1}$

ABSTRACT. A representation theorem is obtained for solutions of the nonlinear functional differential equation

$$
u^{\prime}(t)=F\left(u_{t}\right), \quad t>0, \quad u(t)=\phi(t), \quad t<0,
$$

as a semigroup of nonlinear operators on a space of initial data $X$ of "fading memory type." Equation (1) is studied in the abstract setting of a Banach space $E$. The nonlinear functional $F$ is a uniformly Lipschitz continuous mapping from $X$ to $E$. The semigroup is constructed by transforming (1) to an abstract Cauchy problem

$$
w^{\prime}(t)+A w(t)=0, \quad w(0)=\phi,
$$

in the space $X$ and applying a generation theorem of $M$. Crandall and T. Liggett to the operator $A$ in $X$. The case when (1) is a nonlinear Volterra integrodifferential equation of infinite delay is given special consideration. The semigroup representation is used to obtain finite difference approximations for solutions of (CP) and to study the continuity of solutions of (1) with respect to perturbations of $F$ and $\phi$.

1. Introduction. Our objective is to represent solutions of the initial value problem for the nonlinear functional differential equation

$$
\begin{aligned}
u^{\prime}(t) & =F\left(u_{t}\right), \quad t \geqslant 0, \\
u(t) & =\phi(t), \quad t<0,
\end{aligned}
$$

as a semigroup of nonlinear operators on the space of initial data. Here $u_{t}(x)=u(t+x)$ for $x<0, t>0$.

We will consider (1.1) in the abstract setting of a Banach space $E$ with norm $\|\cdot\|$. The initial data are to be taken from a Banach space $X$ constructed as follows:

Choose $r \in(0, \infty)$ and a positive, nondecreasing function $p$ on $(-\infty,-r]$. Let $X$ be the set of strongly measurable functions $\phi$ from $(-\infty, 0]$ to $E$ such

Presented to the Society, January 23, 1976; received by the editors September 15, 1976.

AMS (MOS) subject classifications (1970). Primary 47H15, 39A40; Secondary 45G99.

Key words and phrases. Nonlinear quasi-contraction semigroup, functional differential equation, Volterra integrodifferential equation, accretive.

'The contents of this paper are a part of the author's Ph.D. thesis written under the direction of Professor John A. Nohel at the University of Wisconsin, Madison. The author wishes to thank Professor Nohel for his concerned guidance and support as well as Professors Michael Crandall and Luc Tartar for suggesting corrections and improvements of a preliminary draft.

- American Mathematical Society 1978 
that $\phi$ is continuous on $[-r, 0]$ and $p \phi$ is integrable on $(-\infty,-r)$. The space $X$ becomes a Banach space under the norm

$$
\|\phi\|_{x}=\sup _{-r<x<0}\|\phi(x)\|+\int_{-\infty}^{-r} p(x)\|\phi(x)\| d x .
$$

We will assume that $F$ is a uniformly Lipschitz continuous mapping from $X$ to $E$, i.e. there is a constant $M>0$ such that

$$
\left\|F\left(\phi_{1}\right)-F\left(\phi_{2}\right)\right\|<M\left\|\phi_{1}-\phi_{2}\right\|_{X} \text { for } \phi_{1}, \phi_{2} \in X .
$$

Under this assumption one has the following classical existence and uniqueness result for solutions of $(1.1)$ on $[0, \infty)$.

LEMMA 1.1. The problem $(1.1)$ has a unique solution $u=u(t ; \phi)$ for every $\phi \in X$ in the sense that

$$
u(t)= \begin{cases}\phi(0)+\int_{0}^{t} F\left(u_{s}\right) d s, & t>0, \\ \phi(t), & t<0 .\end{cases}
$$

This lemma may be proved by standard methods, e.g. Picard iterations or a fixed point argument. We omit the straightforward proof.

Following the construction in [2] for a linear Volterra equation with infinite delay (see Remark 1.5), we define an operator $A$ in $X$ by

$$
A \phi=-\phi^{\prime}, \quad D(A)=\left\{\phi \in X: \phi^{\prime} \in X, \phi^{\prime}(0)=F(\phi)\right\} .
$$

More precisely, by (1.5) we mean that if $\phi \in D(A)$ there exists $\tilde{\phi} \in X$ such that

$$
\begin{aligned}
& \phi(x)=\phi(0)+\int_{0}^{x} \tilde{\phi}(s) d s \text { for a.e. } x \in(-\infty, 0), \\
& \tilde{\phi}(0)=F(\phi) .
\end{aligned}
$$

Then we define $A \dot{\phi}=-\tilde{\phi}$. Note that (1.6) implies that $\phi$ has a strong derivative $\phi^{\prime}$ a.e. and $\phi^{\prime}=\tilde{\phi}$ a.e. (See the appendix of [3] for more details. All integrals are in the sense of Bochner.)

(1.5) is motivated by the fact that the mapping $w(t)=u_{t}$ becomes a solution of the abstract Cauchy problem

$$
\begin{aligned}
w^{\prime}(t)+A w(t) & =0, \quad t>0, \\
w(0) & =\phi,
\end{aligned}
$$

in the sense of a strong solution as defined in [7]. (CP) is the natural setting for applications of nonlinear semigroup theory. It should be observed that $A$ is nonlinear only because of its "nonlinear domain."

The main abstract result of this paper is the following representation 
theorem for solutions of the initial value problem (1.1).

TheOREM 1.2. Let $A$ and $X$ be as defined above and suppose (1.3) holds. Then - A generates a nonlinear quasi-contraction semigroup $S \in Q_{\omega}(X)$ for $\omega>M$ $+p(-r)$. Furthermore, $S$ represents the solution $u=u(t, \phi)$ of $(1.1)$ in the sense that

$$
S(t) \phi=u_{t} \text { for } t>0, \phi \in X .
$$

The notation $S \in Q_{\omega}(C)$ means, in general, that $S$ is a strongly continuous semigroup on a subset $C$ of a Banach space $Y$, and that for some real number $\omega$ satisfies the inequality

$$
\|S(t) x-S(t) y\|_{Y}<e^{\omega t}\|x-y\|_{\gamma} \quad t>0, x, y \in C .
$$

The proof of Theorem 1.2 is carried out in $\$ 2$ by applying the following basic result:

TheOREM 1.3 (M. CRANDALl AND T. Liggett [9]). Let $\omega$ be a real number, $B \in \mathbb{Q}(\omega), \lambda_{0}>0$. If $R(I+\lambda B)$ contains $\overline{D(B)}$ for $0<\lambda<\lambda_{0}$, then $-B$ generates a quasi-contraction semigroup $S \in Q_{\omega}(\overline{D(B)})$.

As in [9] the notation $B \in \mathscr{Q}(\omega)$ means that $B+\omega I$ is accretive. The reader may consult [8] for definitions of the basic terms of semigroup theory used in this paper.

The link we will use between strong solutions of (CP) and the semigroup guaranteed by Theorem 1.3 is the following theorem. This theorem was first proved by H. Brezis and A. Pazy [3] in the case $\omega=0$. It is stated and proved for general real $\omega$ by M. Crandall [8].

Theorem 1.4 (H. Brezis ANd A. Pazy). Let $A$ be closed and satisfy the hypotheses of Theorem 1.3 with $S \in Q_{\omega}(\overline{D(A)})$ generated by $-A$. If (CP) has a strong solution $w$ for some $\phi \in D(A)$, then $w(t)=S(t) \phi$ for $t>0$.

REMARK 1.5. An important special case of (1.1) is the Volterra integrodifferential equation with infinite delay

$$
\begin{cases}u^{\prime}(t)=C u(t)-\int_{-\infty}^{t} a(t-s) g(u(s)) d s, & t \geqslant 0, \\ u(t)=\phi(t), & t \leqslant 0,\end{cases}
$$

where $\phi \in X$ is prescribed and $C$ is a bounded linear operator on $E$. By defining

$$
F(\phi)=C \phi(0)-\int_{-\infty}^{0} a(-s) g(\phi(s)) d s,
$$

one may transform (1.10) to (1.1). It is not difficult to verify that if 
(1.12) $a:(0, \infty) \rightarrow R, a \in L^{1}(0, \infty)$, and $|a(-x)|<K p(x)$ for a.e. $x \in$ $(-\infty,-r)$ and some constant $K>0$; and

(1.13) $g: E \rightarrow E$ and $g$ is uniformly Lipschitz continuous on $E$,

then $F$, given by (1.11) satisfies (1.3). Computation shows that if $g(0)=0$, then $a \in L^{1}(0, \infty)$ may be replaced by $a \in L_{\text {loc }}^{1}(0, \infty)$. Furthermore, one may also take $\{a(t): t>0\}$ to be a family of bounded linear operators on $E$ whose norms satisfy (1.12).

Theorem 1.2 also holds with $X=C_{u}((-\infty, 0] ; E)$, the space of uniformly continuous functions from $(-\infty, 0]$ to $E$ with supremum norm. This space, with $E=R^{n}$, was used by V. Barbu and S. I. Grossman [2] under the additional requirements that the initial functions have a limit at $-\infty$. $A$ semigroup was developed for the linear case of (1.10), i.e. $g(u)=u$. This case is, of course, permitted by our hypotheses although sharper exponential estimates are available from the linear theory under additional necessary hypotheses.

In [17], R. K. Miller uses semigroup techniques to obtain stability results for a class of nonhomogeneous linear Volterra integrodifferential equations.

A particularly interesting case of (1.10) is studied by C. Dafermos in [11] and [12]. Using techniques different from those employed here, existence and asymptotic behaviour results are obtained for (1.10) when $g$ is an unbounded linear differential operator (so that (1.3) does not hold).

The case of (1.10) in which $C$ and $g$ are allowed to be unbounded, nonlinear partial differential operators has been studied by Barbu [1] and by Crandall, Londen, and Nohel [10] by using the theory of monotone operators and leading to results on global existence, boundedness, and asymptotic behaviour of solutions. No nonlinear semigroup theory is directly involved in these results. For the case of (1.10) with $C=0$ and $g$ the subdifferential of a proper, convex, lower semicontinuous function defined on a real Hilbert space, Londen [15], [16] has obtained results on local existence of solutions.

RemarK 1.6. The initial data space $X$ defined by (1.2) is employed by $\mathrm{J}$. Hale in [14] to obtain exponential estimates for solutions of linear functional differential equations with infinite delay. This space is of "fading memory type" and was studied earlier by B. D. Coleman and V. J. Mizel [6]. Hale uses spectral theory to extend and supplement the results of Barbu and Grossman [2] concerning a linear Volterra integrodifferential equation with infinite delay. This setting was discussed in Remark 1.5.

G. F. Webb and C. C. Travis have recently developed semigroup representations for a class of nonlinear functional differential equations with finite delay. In [19] and [20] Webb uses hypotheses and techniques similar to those used here (e.g. Lipschitz continuity of the functional) to obtain a semigroup associated with the solutions of the finite delay analogue of (1.1) with initial 
data spaces $C([-r, 0] ; E)$ and $L^{p}(-r, 0 ; E), p>1$.

H. Flaschka and M. J. Leitman [13] prove directly that the semigroup constructed by Webb in [19] is a classical solution of the corresponding functional differential equation of finite delay. In this paper this link is made for the infinite delay problem (1.1) by using the classical solution of Lemma 1.1 to construct a strong solution of (CP) (Lemma 2.3), then Theorem 1.4 is applied to identify the semigroup with the strong solution of (CP). This method may also be used in the finite delay problem. In general, a semigroup may not be a solution of its corresponding Cauchy problem in a nonreflexive Banach space. See $[9, \S 4]$ for an example.

\section{Proof of Theorem 1.2.}

OUTINE OF PROOF. Assume for the moment that for some fixed $\omega>0$ the following lemmas hold:

LEMMA 2.1. The operator $(I+\lambda(A+\omega I))^{-1}$ is a contraction on $X$ for $\lambda>0$, and there exists $\lambda_{0}>0$ such that $R(I+\lambda A)=X$ for $0<\lambda<\lambda_{0}$.

LEMMA 2.2. The domain of $A$ is dense in $X$.

LEMMA 2.3. The mapping $w:[0, \infty) \rightarrow X$ defined by $w(t)=u_{t}$ is a strong solution of $(\mathrm{CP})$ for $\phi \in D(A)$.

Lemmas 2.1-2.2 imply, by the theorem of Crandall and Liggett (Theorem 1.3) that $-A$ generates a nonlinear semigroup $S \in Q_{\omega}(X)$. The operator $A$ is closed since $m$-associative operators are closed and $A+\omega I$ is $m$-accretive by Lemma 2.1. Therefore, Lemma 2.3 and Theorem 1.4 imply that

$$
S(t) \phi=u_{t} \text { for } \phi \in D(A) \text {. }
$$

We now appeal to the following lemma.

LEMMA 2.4. The mapping $\phi \rightarrow u_{t}(\phi)$, where $u_{t}(x)=u(t+x ; \phi), x<0$, is a continuous mapping from $X$ into $X$ for every $t>0$.

This lemma, together with the continuity of $S(t)$ for every $t>0$ and Lemma 2.2, implies that (2.1) holds for every $\phi \in X$. This completes the proof of the theorem, assuming the truth of the lemmas.

Proof of Lemma 2.1. For reasons that will become clear in the proof, suppose $\omega$ satisfies

$$
\omega>M+p(-r) \text {. }
$$

Choose any $h \in X, \lambda>0$. We wish to find $\phi \in D(A)$ such that

$$
(I+\lambda(A+\omega I)) \phi=h \text {. }
$$

By (1.5) this means that $\phi$ must satisfy

$$
(1+\lambda \omega) \phi(x)-\lambda \phi^{\prime}(x)=h(x), \quad x<0,
$$




$$
(1+\lambda \omega) \phi(0)-\lambda F(\phi)=h(0) \text {. }
$$

By (2.4) $\phi$ must be of the form

$$
\begin{aligned}
\phi(x)= & \phi(0) \exp \left[\left(\omega+\lambda^{-1}\right) x\right] \\
& +\frac{1}{\lambda} \int_{x}^{0} \exp \left[\left(\omega+\frac{1}{\lambda}\right)(x-\eta)\right] h(\eta) d \eta, \quad x<0 .
\end{aligned}
$$

We will first show that $\phi \in X$ for every value of $\phi(0)$. Since $\phi$ is strongly measurable and clearly continuous on $[-r, 0]$ it suffices to show that $\|\phi\|_{X}<$ $\infty$. For $-r<x<0$,

$$
\begin{aligned}
\|\phi(x)\|< & \|\phi(0)\| \exp \left[\left(\omega+\lambda^{-1}\right) x\right] \\
& +\sup _{-r<x<0}\|h(x)\| \frac{1}{1+\lambda \omega}\left(1-\exp \left[\left(\omega+\frac{1}{\lambda}\right) x\right]\right) .
\end{aligned}
$$

Therefore,

(2.7) $\sup _{-r<x<0}\|\phi(x)\|<\max \left(\|\phi(0)\|, \frac{1}{1+\lambda \omega} \sup _{-r<x<0}\|h(x)\|\right)$.

By (2.6), Fubini's Theorem, and the fact that $p$ is nondecreasing,

$$
\begin{aligned}
& \int_{-\infty}^{-r} p(x)\|\phi(x)\| d x<\|\phi(0)\| \int_{-\infty}^{-r} p(x) \exp \left[\left(\omega+\frac{1}{\lambda}\right) x\right] d x \\
& +\frac{1}{\lambda} \int_{-\infty}^{-r} p(x) \int_{x}^{0} \exp \left[\left(\omega+\frac{1}{\lambda}\right)(x-\eta)\right]\|h(\eta)\| d \eta d x \\
& <\frac{\lambda}{1+\lambda \omega}\|\phi(0)\| p(-r) \exp \left[-\left(\omega+\frac{1}{\lambda}\right) r\right] \\
& +\frac{1}{\lambda} \int_{-r}^{0}\|h(\eta)\| \exp \left[-\left(\omega+\frac{1}{\lambda}\right) \eta\right] \\
& \cdot \int_{-\infty}^{-r} p(x) \exp \left[\left(\omega+\frac{1}{\lambda}\right) x\right] d x d \eta \\
& +\frac{1}{\lambda} \int_{-\infty}^{-r}\|h(\eta)\| \exp \left[-\left(\omega+\frac{1}{\lambda}\right) \eta\right] \\
& \cdot \int_{-\infty}^{\eta} p(x) \exp \left[\left(\omega+\frac{1}{\lambda}\right) x\right] d x d \eta \\
& <\frac{\lambda}{1+\lambda \omega}\|\phi(0)\| p(-r) \exp \left[-\left(\omega+\frac{1}{\lambda}\right) r\right] \\
& +\sup _{-r<x<0}\|h(x)\| \frac{p(-r)}{1+\lambda \omega} \int_{-r}^{0} \exp \left[-\left(\omega+\frac{1}{\lambda}\right)(r+\eta)\right] d \eta \\
& +\frac{1}{\lambda} \int_{-\infty}^{-r}\|h(\eta)\| p(\eta) \int_{-\infty}^{\eta} \exp \left[\left(\omega+\frac{1}{\lambda}\right)(x-\eta)\right] d x d \eta \text {. }
\end{aligned}
$$


Therefore we obtain the estimate

$$
\begin{gathered}
\int_{-\infty}^{-r} p(x)\|\phi(x)\| d x<\frac{\lambda}{1+\lambda \omega}\|\phi(0)\| p(-r) \exp \left[-\left(\omega+\frac{1}{\lambda}\right) r\right] \\
+\sup _{-r<x<0}\|h(x)\| \frac{\lambda p(-r)}{(1+\lambda \omega)^{2}}\left(1-\exp \left[-\left(\omega+\frac{1}{\lambda}\right) r\right]\right) \\
+\frac{1}{1+\lambda \omega} \int_{-\infty}^{-r} p(x)\|h(x)\| d x .
\end{gathered}
$$

By (2.7), (2.8), since $h \in X$, we conclude that $\phi \in X$.

Straightforward calculations also show, as expected from (2.4), that

$$
\phi(x)=\phi(0)+\int_{0}^{x} \tilde{\phi}(s) d s
$$

where $\tilde{\phi}=\left(\omega+\lambda^{-1}\right) \phi-\lambda^{-1} h \in X$. It remains to show that (2.5) holds. Substituting (2.6) into (2.5) we see that (2.5) holds if and only if $\phi(0)$ is a fixed point of the map $\Upsilon: E \rightarrow E$ given by

$$
r \theta=h(0) /(1+\lambda \omega)
$$

$$
\begin{aligned}
+\frac{\lambda}{1+\lambda \omega} F\{\theta & \exp \left[\left(\omega+\frac{1}{\lambda}\right) x\right] \\
& \left.+\frac{1}{\lambda} \int_{x}^{0} \exp \left[\left(\omega+\frac{1}{\lambda}\right)(x-\eta)\right] h(\eta) d \eta\right\} .
\end{aligned}
$$

To show that $\Upsilon$ has a (unique) fixed point it suffices to show that $\Upsilon$ is a strict contraction on $E$. By (2.9) and (1.3) we have

$$
\begin{aligned}
\left\|T \theta_{1}-\Upsilon \theta_{2}\right\| & <\frac{\lambda M}{1+\lambda \omega}\left\|\left(\theta_{1}-\theta_{2}\right) \exp \left[\left(\omega+\frac{1}{\lambda}\right) x\right]\right\|_{X} \\
< & \frac{\lambda M}{1+\lambda \omega}\left\|\theta_{1}-\theta_{2}\right\|\left\{\sup _{-r<x<0} \exp \left[\left(\omega+\frac{1}{\lambda}\right) x\right]\right. \\
\left.\quad+\int_{-\infty}^{-r} p(x) \exp \left[\left(\omega+\frac{1}{\lambda}\right) x\right] d x\right\} & <\frac{\lambda M}{1+\lambda \omega}\left\|\theta_{1}-\theta_{2}\right\|\left(1+\frac{\lambda p(-r)}{1+\lambda \omega} \exp \left[-\left(\omega+\frac{1}{\lambda}\right) r\right]\right) \\
< & <\left\|\theta_{1}-\theta_{2}\right\|\left\{\frac{M}{\omega}\left(1+\frac{p(-r)}{\omega} e^{-\omega r}\right)\right\}<\left\|\theta_{1}-\theta_{2}\right\|,
\end{aligned}
$$

where for the last inequality we used a consequence of (2.2). This shows that $\Upsilon$ has a unique fixed point $\theta$. Setting $\phi(0)=\theta$ in (2.6) we obtain (2.3). Hence $R(I+\lambda(A+\omega I))^{-1}=X$ for $\lambda>0$.

Choose $h_{1}, h_{2} \in X$. Let 


$$
\phi_{i}=(I+\lambda(A+\omega I))^{-1} h_{i}, \quad i=1,2 .
$$

Note, by above, that $\phi_{i}$ is given by (2.6) with $\phi_{i}(0)$ the unique fixed point of $\Upsilon$ with $h=h_{i}, i=1,2$. To show that $(I+\lambda(A+\omega I))^{-1}$ is a contraction on $X$ for $\lambda>0$, we show that $\left\|\phi_{1}-\phi_{2}\right\|_{X}<\left\|h_{1}-h_{2}\right\|_{X}$. By the same estimates used to obtain (2.7) and (2.8) we have

$$
\begin{gathered}
\sup _{-r<x<0}\left\|\phi_{1}(x)-\phi_{2}(x)\right\| \\
\quad<\max \left(\left\|\phi_{1}(0)-\phi_{2}(0)\right\|, C /(1+\lambda \omega)\right), \\
\int_{-\infty}^{-r} p(x)\left\|\phi_{1}(x)-\phi_{2}(x)\right\| d x \\
<\frac{\lambda}{1+\lambda \omega}\left\|\phi_{1}(0)-\phi_{2}(0)\right\| p(-r) \exp \left[-\left(\omega+\frac{1}{\lambda}\right) r\right] \\
+\frac{C \lambda p(-r)}{(1+\lambda \omega)^{2}}\left(1-\exp \left[-\left(\omega+\frac{1}{\lambda}\right) r\right]\right) \\
+\frac{1}{1+\lambda \omega} \int_{-\infty}^{-r} p(x)\left\|h_{1}(x)-h_{2}(x)\right\| d x
\end{gathered}
$$

where for brevity we let $C$ denote sup $-r<x<0\left\|h_{1}(x)-h_{2}(x)\right\|$. Therefore, to show that $\left\|\phi_{1}-\phi_{2}\right\|_{X}<\left\|h_{1}-h_{2}\right\|_{X}$, we must obtain a bound on $\| \phi_{1}(0)-$ $\phi_{2}(0) \|$. Since

$$
\phi_{i}(0)=\frac{h_{i}(0)}{1+\lambda \omega}+\frac{\lambda}{1+\lambda \omega} F\left(\phi_{i}\right), \quad i=1,2,
$$

we obtain, using (1.3), that

$$
\left\|\phi_{1}(0)-\phi_{2}(0)\right\|<\frac{C}{1+\lambda \omega}+\frac{\lambda M}{1+\lambda \omega}\left\|\phi_{1}-\phi_{2}\right\|_{X}
$$

Substituting (2.13) in (2.11) and (2.12) we have

$$
\begin{aligned}
\left\|\phi_{1}-\phi_{2}\right\|_{x}< & \frac{C}{1+\lambda \omega}+\frac{\lambda M}{1+\lambda \omega}\left\|\phi_{1}-\phi_{2}\right\|_{x} \\
& +\frac{\lambda}{1+\lambda \omega} p(-r) \exp \left[-\left(\omega+\frac{1}{\lambda}\right) r\right] \\
& \cdot\left(\frac{C}{1+\lambda \omega}+\frac{\lambda M}{1+\lambda \omega}\left\|\phi_{1}-\phi_{2}\right\|_{x}\right) \\
& +\frac{C \lambda p(-r)}{(1+\lambda \omega)^{2}}\left(1-\exp \left[-\left(\omega+\frac{1}{\lambda}\right) r\right]\right) \\
& +\frac{1}{1+\lambda \omega} \int_{-\infty}^{-r} p(x)\left\|h_{1}(x)-h_{2}(x)\right\| d x .
\end{aligned}
$$

Combining terms we obtain 


$$
\begin{aligned}
& \left\{1-\frac{\lambda M}{1+\lambda \omega}\left(1+\frac{\lambda p(-r)}{1+\lambda \omega} \exp \left[-\left(\omega+\frac{1}{\lambda}\right) r\right]\right)\right\}\left\|\phi_{1}-\phi_{2}\right\|_{X} \\
& <C\left(\frac{1}{1+\lambda \omega}+\frac{\lambda p(-r)}{(1+\lambda \omega)^{2}}\right) \\
& \quad+\frac{1}{1+\lambda \omega} \int_{-\infty}^{-r} p(x)\left\|h_{1}(x)-h_{2}(x)\right\| d x
\end{aligned}
$$

By (2.2) we have

$$
\begin{aligned}
\omega & >M+p(-r)\left[\frac{1}{1+\lambda \omega}(\lambda \omega+1)\right] \\
& >M+p(-r)\left[\frac{1}{1+\lambda \omega}\left(\lambda M e^{-\omega r}+1\right)\right] \\
& >M+\frac{\lambda M p(-r)}{1+\lambda \omega} \exp \left[-\left(\omega+\frac{1}{\lambda}\right) r\right]+\frac{p(-r)}{1+\lambda \omega}, \quad \lambda>0 .
\end{aligned}
$$

Multiply both sides of (2.15) by $\lambda /(1+\lambda \omega)$ and rearrange to obtain

$$
\frac{\lambda M}{1+\lambda \omega}\left(1+\frac{\lambda p(-r)}{1+\lambda \omega} \exp \left[-\left(\omega+\frac{1}{\lambda}\right) r\right]\right)<\frac{\lambda \omega}{1+\lambda \omega}-\frac{\lambda p(-r)}{(1+\lambda \omega)^{2}} .
$$

Hence, for every $\lambda>0$,

$$
\begin{gathered}
1-\frac{\lambda M}{1+\lambda \omega}\left(1+\frac{\lambda p(-r)}{1+\lambda \omega} \exp \left[-\left(\omega+\frac{1}{\lambda}\right) r\right]\right) \\
>\frac{1}{1+\lambda \omega}+\frac{\lambda p(-r)}{(1+\lambda \omega)^{2}} .
\end{gathered}
$$

Furthermore, (2.16) gives immediately that for every $\lambda>0$,

$$
1-\frac{\lambda M}{1+\lambda \omega}\left(1+\frac{\lambda p(-r)}{1+\lambda \omega} \exp \left[-\left(\omega+\frac{1}{\lambda}\right) r\right]\right)>\frac{1}{1+\lambda \omega}
$$

Dividing both sides of (2.14) by the coefficient of $\left\|\phi_{1}-\phi_{2}\right\|_{X}$ and using (2.16) and (2.17) we have

$$
\begin{aligned}
\left\|\phi_{1}-\phi_{2}\right\|_{X} & <C+\int_{-\infty}^{-r} p(x)\left\|h_{1}(x)-h_{2}(x)\right\| d x \\
& =\left\|h_{1}-h_{2}\right\|_{X} .
\end{aligned}
$$

To complete the proof note that since $R(I+\lambda A /(1+\lambda \omega))=R(I+\lambda(A$ $+\omega I))=X$ for every $\lambda>0$, we have $R(I+\lambda A)=X$ for $0<\lambda<1 / \omega$.

Proof of LemaMa 2.2. Let $h \in X, \varepsilon>0$ be given. It is a consequence of standard results that we may without loss of generality assume that $h$ is 
continuous with compact support on $(-\infty, 0]$. Choose $\gamma \in(0,1 / \omega]$ and define

$$
\phi_{\lambda}=(I+\lambda A)^{-1} h \text { for } 0<\lambda<\gamma .
$$

As in (2.6) with $\omega=0$ this means that

$$
\phi_{\lambda}(x)=\theta_{\lambda} e^{x / \lambda}+\frac{1}{\lambda} \int_{x}^{0} \exp \left[\frac{1}{\lambda}(x-\eta)\right] h(\eta) d \eta, \quad x<0,
$$

where $\theta_{\lambda}=\phi_{\lambda}(0)$ satisfies

$$
\theta_{\lambda}=\lambda F\left(\phi_{\lambda}\right)+h(0) \text {. }
$$

By the same estimates used to obtain (2.7) and (2.8), except with $\omega=0$, there is a constant $C$ independent of $\lambda$ such that

$$
\left\|\phi_{\lambda}\right\|_{x}<C\left(\left\|\theta_{\lambda}\right\|+\|h\|_{x}\right), \quad 0<\lambda<\gamma .
$$

Note that by (1.3), (2.20), and (2.21),

$$
\begin{aligned}
\left\|\theta_{\lambda}-h(0)\right\| & <\lambda\left\|F\left(\phi_{\lambda}\right)\right\|<\lambda\left(M\left\|\phi_{\lambda}\right\|_{x}+\|F(0)\|\right) \\
& <\lambda M C\left\|\theta_{\lambda}-h(0)\right\|+\lambda\left\{M C\left(\|h(0)\|+\|h\|_{X}\right)+\|F(0)\|\right\} .
\end{aligned}
$$

Hence

$$
\left\|\theta_{\lambda}-h(0)\right\| \rightarrow 0 \text { as } \lambda \rightarrow 0 \text {. }
$$

We now need a result which has some interest in its own right when considering (1.1) with initial data which are uniformly continuous.

Claim. If $h$ is uniformly continuous and $\phi_{\lambda}$ is defined by (2.18), then

$$
\left\|\phi_{\lambda}(x)-h(x)\right\| \rightarrow 0 \text { as } \lambda \rightarrow 0
$$

uniformly for $x<0$.

Proof of Clam. By (2.19),

$$
\begin{aligned}
\phi_{\lambda}(x)-h(x)= & e^{x / \lambda}\left(\theta_{\lambda}-h(x)\right) \\
& +\frac{1}{\lambda} \int_{x}^{0} \exp \left[\frac{1}{\lambda}(x-\eta)\right](h(\eta)-h(x)) d \eta .
\end{aligned}
$$

Choose $\delta>0$. Let $\varepsilon(\delta)=\sup _{|x-\eta|<\delta}\|h(\eta)-h(x)\|$ so that $\varepsilon(\delta) \rightarrow 0$ as $\delta \rightarrow 0$. By (2.23) it is easy to see that

$$
\left\|\phi_{\lambda}(x)-h(x)\right\|<\left\|\theta_{\lambda}-h(0)\right\|+2 \varepsilon(\delta)
$$

for $-\delta<x<0$. For $-\infty<x<-\delta$ we obtain, again from (2.23), 


$$
\begin{aligned}
\left\|\phi_{\lambda}(x)-h(x)\right\|< & e^{-\delta / \lambda}\left(\left\|\theta_{\lambda}\right\|+\|h\|_{\infty}\right) \\
& +\frac{1}{\lambda} \int_{x}^{x+\delta} \exp \left[\frac{1}{\lambda}(x-\eta)\right]\|h(\eta)-h(x)\| d \eta \\
& +\frac{1}{\lambda} \int_{x+\delta}^{0} \exp \left[\frac{1}{\lambda}(x-\eta)\right]\|h(\eta)-h(x)\| d \eta \\
< & e^{-\delta / \lambda}\left(\left\|\theta_{\lambda}\right\|+\|h\|_{\infty}\right)+\varepsilon(\delta)+2\|h\|_{\infty} e^{-\delta / \lambda}
\end{aligned}
$$

Combining these estimates proves (*).

Now returning to the proof of the lemma, we have from the definition of the norm in $X$ that

$$
\begin{gathered}
\left\|\phi_{\lambda}-h\right\|_{X}<\left\|\phi_{\lambda}-h\right\|_{\infty}\left(1+\int_{-T}^{-r} p(x) d x\right) \\
+\int_{-\infty}^{-T} p(x)\left\|\phi_{\lambda}(x)\right\| d x,
\end{gathered}
$$

where $T>r$ is chosen so that $h(x)=0$ for $x<-T$. For $x<-T$, by (2.19) we have

$$
\begin{aligned}
\left\|\phi_{\lambda}(x)\right\| & <\left\|\theta_{\lambda}\right\| e^{x / \lambda}+\frac{1}{\lambda} \int_{-T}^{0} \exp \left[\frac{1}{\lambda}(x-\eta)\right]\|h(\eta)\| d \eta \\
& <C \exp \left[\frac{1}{\lambda}(x+T)\right],
\end{aligned}
$$

where $C$ is a constant independent of $\lambda$. Therefore,

$$
\begin{aligned}
\int_{-\infty}^{-T} p(x)\left\|\phi_{\lambda}(x)\right\| d x & <C p(-T) \int_{-\infty}^{-T} \exp \left[\frac{1}{\lambda}(x+T)\right] d x \\
& =\lambda C p(-T) .
\end{aligned}
$$

This estimate together with (2.26) and (*) completes the proof of Lemma 2.2 since $\phi_{\lambda} \in D(A)$ for $0<\lambda<\gamma$.

Proof of Lemma 2.3. Choose $\phi \in D(A)$ and let $u(t)=u(t ; \phi)$ be the unique solution of (1.1) satisfying (1.4). Let $w(t)=u_{t}$ where $u_{t}(x)=u(t+x$; $\phi)$ for $x<0$. We will first prove that $u_{t} \in X$ for $t>0$. It suffices to show that $\left\|u_{i}\right\|_{X}$ is finite. Employing a change of variable and using the hypothesis that $p$ is nondecreasing we have

$$
\begin{aligned}
\left\|u_{t}\right\|_{X}< & \sup _{t-r<x<t}\|u(x)\|+\sup _{-r<x<t-r}\|u(x)\| \int_{-r}^{t-r} p(x) d x \\
& +\int_{-\infty}^{-r} p(x)\|\phi(x)\| d x
\end{aligned}
$$

which is finite since $\phi \in X$ and $u$ is bounded on $[-r, t]$.

For brevity define 


$$
q(s)=F\left(u_{s}\right), \quad s>0 .
$$

Then by (1.4),

$$
u_{t}(x)= \begin{cases}\phi(0)+\int_{0}^{t+x} q(s) d s, & -t<x<0, \\ \phi(t+x), & -\infty<x<-t .\end{cases}
$$

Next we show that the mapping $t \rightarrow u_{t}$ from $[0, \infty)$ to $X$ is continuous. Choose $t>s>0$ and let $h=t-s$. Then

$$
\begin{aligned}
\left\|u_{t}-u_{s}\right\|_{X}< & \sup _{-r<x<t}\|u(x)-u(x-h)\| \\
& \cdot\left(1+\int_{-r}^{t-r} p(x-t) d x\right) \\
& +\int_{-\infty}^{-r} p(x)\|\phi(x)-\phi(x-h)\| d x .
\end{aligned}
$$

Since $u$ is continuous on the bounded interval $[-r, t]$, the first term goes to zero as $h \rightarrow 0$. Since $\phi \in X$, the second term goes to zero as $h \rightarrow 0$ by a standard argument. This shows that $w(t)=u_{t} \in C([0, \infty) ; X)$.

We now show that $u_{t} \in D(A)$ for $t>0$. Since $\phi \in D(A)$, there exists $\tilde{\phi} \in X$ such that

$$
\begin{aligned}
& \phi(x)=\phi(0)+\int_{0}^{x} \tilde{\phi}(s) d s, \quad x<0, \\
& \tilde{\phi}(0)=F(\phi)=q(0) .
\end{aligned}
$$

Hence by (2.29) and (2.31),

$$
u_{t}(x)= \begin{cases}\phi(0)+\int_{0}^{t+x} q(s) d s, & -t<x<0, \\ \phi(0)+\int_{0}^{t+x} \tilde{\phi}(s) d s, & -\infty<x<-t .\end{cases}
$$

Using (2.33) and $\phi(0)=u(t)-\int_{0}^{t} q(s) d s$, we have

$$
u_{t}(x)=\left\{\begin{array}{l}
u(t)+\int_{t}^{t+x} q(s) d s, \quad-t<x<0, \\
u(t)-\int_{0}^{t} q(s) d s+\int_{0}^{t+x} \tilde{\phi}(s) d s, \quad-\infty<x<-t .
\end{array}\right.
$$

Define a function on $(-\infty,+\infty)$ by

$$
\gamma(s)= \begin{cases}q(s), & s>0 \\ \tilde{\phi}(s), & s<0\end{cases}
$$


Since $q$ is continuous on $[0, \infty), \tilde{\phi}$ is continuous on $[-r, 0]$, and $q(0)=\tilde{\phi}(0)$ by (2.32), we have that $\gamma$ is continuous on $[-r, \infty)$. Therefore, by arguments similar to those used earlier, it is easy to see that $\gamma_{t} \in X$ for $t>0$. By (2.34),

$$
\begin{aligned}
u_{t}(x) & =u(t)+\int_{t}^{t+x} \gamma(s) d s \\
& =u_{t}(0)+\int_{0}^{x} \gamma_{t}(s) d s \text { for }-\infty<x<0 .
\end{aligned}
$$

Furthermore,

$$
\gamma_{t}(0)=\gamma(t)=q(t)=F\left(u_{t}\right)
$$

Hence (2.35) and (2.36) show that $u_{t} \in D(A)$ for $t>0$.

Next we show that $u_{t} \in W_{\mathrm{loc}}^{1,1}((0, \infty) ; X)$ for $t>0$. This means to show that

$$
u_{t}-u_{s}=\int_{s}^{t} \gamma_{\tau} d \tau, \quad t, s \geq 0 .
$$

Note that $v(t)=\gamma_{t} \in L_{\mathrm{loc}}^{1}(0, \infty ; X)$, since by estimates of the type used to show that $\left\|u_{t}\right\|_{X}$ is finite, it is not difficult to see that $\left\|\gamma_{t}\right\|_{X}$ is bounded uniformly on bounded $t$ intervals. Assume, without loss of generality, that $s>t>0$. By (2.35),

$$
u(s+x)-u(s)=\int_{0}^{x} \gamma(s+\tau) d \tau, \quad s>0, x<0 .
$$

Let $x=t-s+y, y<0$, in (2.38) to obtain

$$
u(t+y)-u(s)=\int_{0}^{t-s+y} \gamma(s+\tau) d \tau=\int_{s}^{t+y} \gamma(\tau) d \tau .
$$

It also follows from (2.38) that

$$
-u(s)=\int_{0}^{y} \gamma(s+\tau) d \tau-u(s+y)
$$

Combining (2.39) and (2.40) we have

$$
\begin{aligned}
u(t+y)-u(s+y) & =\int_{s}^{t+y} \gamma(\tau) d \tau-\int_{s}^{s+y} \gamma(\tau) d \tau \\
& =\int_{s+y}^{t+y} \gamma(\tau) d \tau=\int_{s}^{t} \gamma(\tau+y) d \tau \\
& =\int_{s}^{t} \gamma_{\tau}(y) d \tau, \quad y<0 .
\end{aligned}
$$

(2.41) proves (2.37).

Finally we show that $w(t)=u_{t}$ satisfies (CP) a.e. when $A$ is defined by (1.5). By (2.35), $A u_{t}=-\gamma_{t}$ for $t>0$. By (2.37),

$$
d u_{t} / d t=\gamma_{t} \text { for a.e. } t \in(0, \infty) \text {. }
$$


Clearly $w(0)=u_{0}=\phi$ by definition and, by (2.42),

$$
d u_{t} / d t+A u_{t}=0 \text { for a.e. } t \in(0, \infty) \text {. }
$$

This completes the proof of Lemma 2.3.

Proof of Lemma 2.4. Choose $\phi, \psi \in X$. Let $u(t)=u(t ; \phi)$ and $v(t)=u(t$; ४). By (1.4) and (1.3),

$$
\|u(t)-v(t)\|<\|\phi(0)-\psi(0)\|+M \int_{0}^{t}\left\|u_{s}-v_{s}\right\|_{X} d x .
$$

Using the definition of the norm in $X$ we obtain

$$
\begin{aligned}
\left\|u_{s}-v_{s}\right\|_{X}= & \sup _{-r<x<0}\|u(s+x)-v(s+x)\| \\
& +\int_{-\infty}^{-r} p(x)\|u(s+x)-v(s+x)\| d x .
\end{aligned}
$$

Using a change of variable and the fact that $p$ is nondecreasing we obtain

$$
\begin{aligned}
\left\|u_{s}-v_{s}\right\|_{X}< & \sup _{-r<x<s}\|u(x)-v(x)\| \\
& +\int_{-r}^{-r+s} p(x-s)\|u(x)-v(x)\| d x \\
& +\int_{-\infty}^{-r} p(x-s)\|u(x)-v(x)\| d x \\
< & (1+s p(-r))\left(\sup _{-r<x<0}\|\phi(x)-\psi(x)\|\right. \\
& +\int_{-\infty}^{-r} p(x)\|\phi(x)-\psi(x)\| d x \\
< & (1+\operatorname{sp}(-r))\|\phi-\psi\|_{x} \\
& +(1+s p(-r)) \sup _{0<x<s}\|u(x)-v(x)\| .
\end{aligned}
$$

Using (2.43) in (2.44) we obtain

$$
\left\|u_{s}-v_{s}\right\|_{X}<2 P(s)\|\phi-\psi\|_{X}+M P(s) \int_{0}^{s}\left\|u_{\tau}-v_{\tau}\right\|_{X}, d \tau,
$$

where $P(s)=(1+s p(-r))$. A straightforward application of Gronwall's inequality now yields

$$
\begin{aligned}
\left\|u_{s}-v_{s}\right\|_{X} & <\|\phi-\psi\|_{X}\left\{2 P(s)+2 M P(s)\left(\int_{0}^{s} P(\sigma) d \sigma\right) \exp \left[M \int_{0}^{s} P(\sigma) d \sigma\right]\right\} \\
& <\|\phi-\psi\|_{X}\left\{2 P(s)+2 M s P^{2}(s) \exp [s M P(s)]\right\}, \quad s>0,
\end{aligned}
$$


since $P$ is increasing. This estimate completes the proof of Lemma 2.4. This also completes the proof of Theorem 1.2.

\section{Two applications of the semigroup representation.}

Remark 3.1. By Lemma 2.1 there exists $\lambda_{0}>0$ such that $(I+\lambda A)^{-1}$ exists as a continuous operator on $X$ for $0<\lambda<\lambda_{0}$. By the analysis of Lemma 2.1 with $\omega=0$, it is easy to see that $\phi=(I+\lambda A)^{-1} h$ is given by

$$
\phi(x)=\phi(0) e^{x / \lambda}+\frac{1}{\lambda} \int_{x}^{0} \exp \left[\frac{1}{\lambda}(x-\eta)\right] h(\eta) d \eta
$$

for $x<0,0<\lambda<\lambda_{0}, h \in X$, where $\phi(0)$ is the fixed point of the map

$$
r \theta=h(0)+\lambda F\left(\theta e^{x / \lambda}+\frac{1}{\lambda} \int_{x}^{0} \exp \left[\frac{1}{\lambda}(x-\eta)\right] h(\eta) d \eta\right) .
$$

Therefore $S_{n}(t)=(I+t A / n)^{-n}$ exists as a continuous operator on $X$ for $0<t<n \lambda_{0}, n=0,1,2, \ldots$ By Theorem 1.2 the family of operators $S_{n}(t)$ converges in the strong sense to the semigroup $S(t)$ which represents solutions of (1.1). The operators $S_{n}(t)$ are finite difference approximations of the solutions of (CP). See [7] for a more detailed discussion.

To compare the operators $S_{n}(t)$ we note first that

$$
\begin{aligned}
S_{n+1}(t) & =\left(I+\frac{t}{n+1} A\right)^{-1}\left(I+\frac{t}{n+1} A\right)^{-n} \\
& =\left(I+\frac{t}{n+1} A\right)^{-1} S_{n}(\tilde{t})
\end{aligned}
$$

where $i=n t /(n+1), 0<t<(n+1) \lambda_{0}, n=0,1,2, \ldots$, and $S_{0}(0)=I$. Combining (3.1)-(3.3) we obtain

$$
\begin{aligned}
\left(S_{n+1}(t) h\right)(x)= & \left(S_{n+1}(t) h\right)(0) \exp \left(\frac{n+1}{t} x\right) \\
& +\frac{n+1}{t} \int_{x}^{0} \exp \left[\frac{n+1}{t}(x-\eta)\right]\left(S_{n}(\tilde{t}) h\right)(\eta) d \eta,
\end{aligned}
$$

where $\left(S_{n+1}(t) h\right)(0)$ is the fixed point of the mapping

$$
\begin{aligned}
\mathrm{T}_{n} \theta=\left(S_{n}(\tilde{t}) h\right)(0) & \\
+\frac{t}{n+1} F\{ & \theta \exp \left(\frac{n+1}{t} x\right) \\
& \left.+\frac{n+1}{t} \int_{x}^{0} \exp \left[\frac{n+1}{t}(x-\eta)\right]\left(S_{n}(\tilde{t}) h\right)(\eta) d \eta\right\},
\end{aligned}
$$

and $\tilde{t}=n t /(n+1), 0<t<(n+1) \lambda_{0}, h \in X, x<0, n=0,1,2, \ldots, S_{0}(0)$ = I. 
RemarK 3.2. As another application of Theorem 1.2 we obtain the following result concerning the continuity of solutions with respect to changes in $F$ and $\phi$.

COROLLARY 3.1. Let $\rho_{0}>0$; let $u^{\rho}$ denote the solution of

$$
\begin{aligned}
\left(u^{\rho}\right)^{\prime}(t) & =F^{\rho}\left(\left(u^{\rho}\right)_{t}\right), \quad t>0, \\
u^{\rho}(t) & =\phi^{\rho}(t), \quad t<0,
\end{aligned}
$$

for $0<\rho<\rho_{0}$. We assume that there exists a constant $M$ independent of $\rho$ such that

$$
\left\|F^{\rho}\left(\phi_{1}\right)-F^{\rho}\left(\phi_{2}\right)\right\|<M\left\|\phi_{1}-\phi_{2}\right\|_{X}
$$

for $0<\rho<\rho_{00} \phi_{1}, \phi_{2} \in X$,

$$
\left\|F^{\rho}(\phi)-F^{0}(\phi)\right\| \rightarrow 0 \text { as } \rho \rightarrow 0 \text { for } \phi \in X,
$$

and

$$
\left\|\phi^{\rho}-\phi^{0}\right\|_{X} \rightarrow 0 \text { as } \rho \rightarrow 0 .
$$

Then $u^{\rho}\left(t ; \phi^{\rho}\right) \rightarrow u^{0}\left(t ; \phi^{9}\right)$ as $\rho \rightarrow 0$ uniformly on bounded subsets of $[0, \infty)$ for every $\phi \in X$.

Proof. We will use Theorem 1.2 to apply the following result of nonlinear semigroup theory.

TheORem 3.2 (H. Brezis AND A. PAZY [5]). Let $A^{\rho} \in \mathbb{Q}\left(\omega_{\rho}\right), 0<\rho<\rho_{0}$, such that $R\left(I+\lambda A^{\rho}\right)$ contains $\overline{D\left(A^{\rho}\right)}$ for $0<\lambda<\lambda_{0}, 0<\rho<\rho_{0}$. Let $S^{\rho}(t)$ be the semigroup generated by $-A^{P}$. If

(i) $0<\omega_{\rho}<\alpha<\infty$ for $0<\rho<\rho_{00}$

(ii) $\lim _{\rho \rightarrow 0}\left(I+\lambda A^{\rho}\right)^{-1} h=\left(I+\lambda A^{9}\right)^{-1} h$ for every $h \in \bar{D}$ and $0<\lambda<\lambda_{0}$, where $D=\cap_{\rho>0} \overline{D\left(A^{\rho}\right)} \cap D\left(A^{9}\right)$, then

(iii) $\lim _{\rho \rightarrow 0} S^{\rho}(t) \psi=S^{0}(t) \psi$ for every $\psi \in \bar{D}$, and the limit is uniform on bounded $t$ intervals.

To apply this theorem, let $A^{P} \psi=-\psi^{\prime}$ with

$$
D\left(A^{\rho}\right)=\left\{\psi \in X: \psi^{\prime} \in X, \psi^{\prime}(0)=F^{\rho}(\psi)\right\}, \quad 0<\rho<\rho_{0} .
$$

Then by Theorem 1.2 and (3.5),

$$
A^{\rho} \in \mathbb{Q}(M+p(-r)), \quad 0<\rho<\rho_{0},
$$

and

$$
R\left(I+\lambda A^{\rho}\right)=X=\overline{D\left(A^{\rho}\right)} \text { for } 0<\lambda<(M+p(-r))^{-1} .
$$

Furthermore, $-A^{\rho}$ generates a semigroup $S^{\rho}(t)$ on $X$ satisfying

$$
\left(S^{\rho}(t) \psi\right)(x)=u^{\rho}(t+x ; \psi) \text { for } t>0, x<0,
$$

and for every $\psi \in X, 0<\rho<\rho_{0}$. We have that (i) holds with $\alpha=M+$ 
$p(-r)$. It remains to show that (ii) holds. Note that $\bar{D}=X$. Choose $\lambda_{0}<1$ and $\lambda_{0}<(2 M(1+p(-r)))^{-1}$. Fix $h \in X$ and $0<\lambda<\lambda_{0}$. Let $\psi_{\rho}=(I+$ $\left.\lambda A^{\rho}\right)^{-1} h$. We know from the proof of Lemma 2.1 that

$$
\psi_{\rho}(x)=\psi_{\rho}(0) e^{x / \lambda}+\frac{1}{\lambda} \int_{x}^{0} \exp \left[\frac{1}{\lambda}(x-\eta)\right] h(\eta) d \eta,
$$

where $\psi_{\rho}(0)$ satisfies

$$
\psi_{\rho}(0)=h(0)+\lambda F^{\rho}\left(\psi_{\rho}\right), \quad 0<\rho<\rho_{0} .
$$

Let $\varepsilon>0$ be given. By (3.6) there exists $\delta>0$ such that

$$
\left\|F^{\rho}\left(\psi_{0}\right)-F^{0}\left(\psi_{0}\right)\right\|<\varepsilon \text { for } 0<\rho<\delta .
$$

Hence by (3.9),

$$
\begin{aligned}
\left\|\psi_{\rho}(0)-\psi_{0}(0)\right\| & <\lambda\left\|F^{\rho}\left(\psi_{\rho}\right)-F^{0}\left(\psi_{0}\right)\right\| \\
& <\lambda\left\|F^{\rho}\left(\psi_{\rho}\right)-F^{\rho}\left(\psi_{0}\right)\right\|+\lambda\left\|F^{\rho}\left(\psi_{0}\right)-F^{0}\left(\psi_{0}\right)\right\| .
\end{aligned}
$$

Therefore using (3.5) we obtain

$$
\left\|\psi_{\rho}(0)-\psi_{0}(0)\right\|<\lambda M\left\|\psi_{\rho}-\psi_{0}\right\|_{X}+\lambda \varepsilon, \quad 0<\rho<\delta .
$$

By (3.8),

so that

$$
\psi_{\rho}(x)-\psi_{0}(x)=\left(\psi_{\rho}(0)-\psi_{0}(0)\right) e^{x / \lambda}
$$

$$
\begin{aligned}
\left\|\psi_{\rho}-\psi_{0}\right\|_{X}= & \sup _{-r<x<0}\left\|\psi_{\rho}(0)-\psi_{0}(0)\right\| e^{x / \lambda} \\
& +\int_{-\infty}^{-r} p(x)\left\|\psi_{\rho}(0)-\psi_{0}(0)\right\| e^{x / \lambda} d x \\
& <\left\|\psi_{\rho}(0)-\psi_{0}(0)\right\|\left(1+p(-r) \int_{-\infty}^{-r} e^{x / \lambda} d x\right) \\
& <\left\|\psi_{\rho}(0)-\psi_{0}(0)\right\|(1+p(-r)) .
\end{aligned}
$$

So combining (3.10) and (3.11) we obtain

$$
\begin{aligned}
\left\|\psi_{\rho}-\psi_{0}\right\|_{X} & <\lambda M(1+p(-r))\left\|\psi_{\rho}-\psi_{0}\right\|_{X}+\lambda_{\varepsilon}(1+p(-r)) \\
& <\frac{1}{2}\left\|\psi_{\rho}-\psi_{0}\right\|_{X}+\lambda_{0} \varepsilon(1+p(-r)) .
\end{aligned}
$$

Therefore

$$
\left\|\psi_{\rho}-\psi_{0}\right\|_{X}<2 \lambda_{0} \varepsilon(1+p(-r)) \text { for } 0<\rho<\delta, 0<\lambda<\lambda_{0} .
$$

Since $\varepsilon>0$ was arbitrary, this proves that

$$
\left\|\psi_{\rho}-\psi_{0}\right\|_{X} \rightarrow 0 \text { as } \rho \rightarrow 0
$$

and so (ii) holds.

The conclusion of Theorem 3.2 then tells us that 


$$
\left\|S^{\rho}(t) \psi-S^{0}(t) \psi\right\|_{X} \rightarrow 0 \text { as } \rho \rightarrow 0 \text { for } \psi \in X
$$

uniformly on bounded $t$ intervals. Therefore, by (3.5), (3.7), and (3.12),

$$
\begin{aligned}
\left\|S^{\rho}(t) \phi^{\rho}-S^{0}(t) \phi^{0}\right\|_{X} & <\left\|S^{\rho}(t) \phi^{\rho}-S^{\rho}(t) \phi^{0}\right\|_{X}+\left\|S^{\rho}(t) \phi^{0}-S^{0}(t) \phi^{0}\right\|_{X} \\
& <M\left\|\phi^{\rho}-\phi^{0}\right\|_{X}+\left\|S^{\rho}(t) \phi^{0}-S^{0}(t) \phi^{0}\right\|_{X}
\end{aligned}
$$

which goes to zero as $\rho \rightarrow 0$ uniformly on bounded $t$ intervals. In particular,

$$
\sup _{-r<x<0}\left\|u^{\rho}\left(t+x ; \phi^{\rho}\right)-u^{0}\left(t+x ; \phi^{0}\right)\right\| \rightarrow 0 \text { as } \rho \rightarrow 0
$$

uniformly on bounded $t$ intervals. This completes the proof of Corollary 3.1 .

\section{REFERENCES}

1. V. Barbu, Integrodifferential equations in Hilbert spaces, An. Sti. Univ. "Al. I. Cuza" Iasi Sect. I 19 (1973), 365-383.

2. V. Barbu and S. I. Grossman, Asymptotic behavior of linear integrodifferential systems, Trans. Amer. Math. Soc. 173 (1972), 277-288. MR 46 \# 7826.

3. H. Brézis, Opérateurs maximaux monotones et semi-groupes de contractions dans les espaces de Hilbert, Math. Studies, No. 5, North-Holland, Amsterdam, 1973. MR 50 \# 1060.

4. H. Brézis and A. Pazy, Accretive sets and differential equations in Banach spaces, Israel J. Math. 8(1970), 367-383. MR 43 \# 1000.

5. Convergence and approximation of semigroups of nonlinear operators in Banach spaces, J. Functional Analysis 9 (1972), 63-74. MR 45 \#2529.

6. B. D. Coleman and V. J. Mizel, On the stability of solutions of functional-differential equations, Arch. Rational Mech. Anal. 30 (1968), 173-196. MR 37 \#5499.

7. M. G. Crandall, An introduction to evolution governed by accretive operators, Proc. Internat. Sympos. Dynamical Systems (Brown Univ., Providence, R. I., 1974).

8. - Semigroups of nonlinear transformations in Banach spaces, Contributions to Nonlinear Functional Analysis (E. Zarantonello, editors), Academic Press, New York, 1971, pp. 157-179.

9. M. G. Crandall and T. M. Liggett, Generation of semi-groups of nonlinear transformations on general Banach spaces, Amer. J. Math. 93 (1971), 265-298. MR 44 \#4563.

10. M. G. Crandall, S. O. Londen and J. A. Nohel, An abstract nonlinear Volterra integradifferential equation (to appear); See also Notices Amer. Math. Soc. 23 (1976), A-146. Abstract \#731-45-4.

11. C. M. Dafermos, An abstract Volterra equation with applications to linear viscoelasticity, J. Differential Equations 7 (1970), 554-569. MR 41 \#4305.

12. Asymptotic stability in viscoelasticity, Arch. Rational Mech. Anal. 37 (1970), 297-308. MR 43 \#7117.

13. H. Flaschka and M. J. Leitman, On semigroups of nonlinear operators and the solutions of the functional differential equation $\dot{x}(t)=F\left(x_{t}\right)$, J. Math. Anal. Appl. 49 (1975), 649-658.

14. J. K. Hale, Functional differential equations with infinite delays, J. Math. Anal. Appl. 48 (1974), 276-283. MR 51 \# 1067.

15. S. O. Londen, On a Volterra equation in Banach space, MRC Tech. Summary Report \# 1558, Univ. of Wisconsin, Nov. 1975.

16. Some local existence results on an integral equation in a Banach space, MRC Tech. Summary Report \# 1556, Univ. of Wisconsin, Nov. 1975.

17. R. K. Miller, Linear Volterra integrodifferential equations as semigroups, Funkcial. Ekvac. 17 (1974), 39-55. MR 50 \#3003. 
18. C. C. Travis and G. F. Webb, Existence and stability for partial functional differential equations, Trans. Amer. Math. Soc. 200 (1974), 395-418.

19. G. F. Webb, Autonomous nonlinear functional differential equations and nonlinear semigroups, J. Math. Anal. Appl. 46 (1974), 1-12. MR 50 \# 722.

20. - Functional differential equations and nonlinear semigroups in $L^{P}$ spaces, $J$. Differential Equations 20 (1976), 71-89.

Departmant of Mathematics, University of ARKansas, FayetTeViLle, Arkansas 72701 